\title{
Perfil dos pacientes internados em serviços de pediatria no município do Rio de Janeiro: mudamos?
}

\author{
I 1 Josélia Giordano Duarte, 2 Saint Clair Gomes, ${ }^{3}$ Marcia \\ Teixeira Pinto, ${ }^{4}$ Maria Auxiliadora S. Mendes Gomes I
}

Resumo: Apesar das alterações observadas nos últimos anos no adoecimento e na mortalidade na infância com marcada redução das internaçôes por doenças infectocontagiosas, existem poucos estudos que apresentem o perfil clínico e demográfico das internações pediátricas em nosso meio. $\mathrm{O}$ estudo teve como objetivo identificar as características e os procedimentos diagnósticos e terapêuticos das internações pediátricas em hospitais do sistema público de saúde no município do Rio de Janeiro. Quanto ao método, foi realizado estudo retrospectivo desenvolvido em quatro hospitais públicos no Rio de Janeiro, em amostra constituída de 170 internações ocorridas nas enfermarias de pediatria no período de janeiro a dezembro de 2008. As informações obtidas evidenciaram elevada frequência de crianças portadoras de doenças crônicas (47.6\%) no conjunto das internações estudadas. Os serviços também apresentaram elevado percentual de pacientes com histórico de reinternação (35.3\%). A análise por unidade mostrou que, entre os quatro serviços estudados, apenas um apresentou menor proporção de crianças portadoras de doenças crônicas e menor frequência de reinternações. Nas outras três unidades hospitalares, em mais da metade do grupo estudado, as causas de internaçôes encontradas estavam ligadas às doenças crônicas. Os resultados confirmam a necessidade de organização dos serviços pediátricos no sentido de estarem preparados, com estrutura e processos de cuidado adequados, para o manejo, tratamento e acompanhamento do paciente portador de doença crônica.

> Palavras-chave: internação hospitalar; assistência pediátrica; doenças crônicas na infância.

\author{
1 Departamento Geral de \\ Hospitais do Ministério da \\ Saúde no Rio de Janeiro. \\ Endereço eletrônico: \\ jghduarte@hotmail.com \\ 2 Unidade de Pesquisa Clínica \\ (IFF/FIOCRUZ) Endereço \\ eletrônico: scgomes@iff. \\ fiocruz.br \\ ${ }^{3}$ Núcleo de Avaliação de \\ Tecnologias em Saúde (IFF / \\ FIOCRUZ). Endereço eletrônico: \\ mpinto@iff.fiocruz.br \\ ${ }_{4}^{4}$ Pós-Graduação em Saúde da \\ Criança e da Mulher, Núcleo de \\ Avaliação de Tecnologias em \\ Saúde (IFF / FIOCRUZ). Endereço \\ eletrônico: maria@iff.fiocruz.br
}




\section{Introdução}

O perfil de morbimortalidade na infância e na adolescência vem passando por um profundo processo de transição. No Brasil, assim como em outros países em desenvolvimento, a assistência hospitalar pediátrica, até bem poucos anos atrás, era voltada para o tratamento de doenças agudas como diarreia, verminoses, doenças respiratórias, e outras doenças infecciosas em geral associadas a níveis variados de desnutrição (ROCHA et al., 1997).

Vários fatores foram responsáveis pela mudança nesse quadro, que foi acompanhado também pela redução das taxas de mortalidade infantil no país. A disponibilidade de programas de saúde que ampliaram o acesso e o conhecimento acerca da imunização, aleitamento materno e a reidratação oral, associados às mudanças na dinâmica social, contribuíram para a redução observada na mortalidade de crianças no primeiro ano de vida (VICTORA, 2001; JOBIN; AERTS, 2008; VICTORA, 2009; VOLPE, 2009).

As mudanças no perfil de internações pediátricas também decorrem dos processos de incorporação de tecnologias que propiciam uma sobrevida maior às crianças que antes estavam destinadas a morrer precocemente (VARGAS et al.,1994, LEJARRAGA, 2006). Hewson (1999) em estudo realizado na Austrália, encontrou a frequência de cerca de $50 \%$ de doenças crônicas nas consultas pediátricas. Esses autores destacam a crescente prevalência da doença crônica na infância, definida por Silva (2001) como uma desordem que tem uma base biológica, psicológica ou cognitiva, e que produz limitação de função ou atividade, dependência de medicação ou dieta especial, ou ainda necessidade de cuidados médicos especiais.

A atenção requerida por crianças e adolescentes portadores de doenças crônicas também tem sido objeto de crescente interesse (MATLOW, 2006). Muitas dessas crianças apresentam sequelas, seja pela condição de risco a que foram expostas ou em consequência dos procedimentos médicos a que foram submetidas durante o tratamento (VARGAS et al.,1994).

As mudanças descritas condicionam novos desafios para a organização dos serviços assistenciais, incluindo a necessidade de produção de conhecimento no campo da gestão hospitalar que oriente o estabelecimento das prioridades nos serviços de pediatria e o planejamento dos programas de qualificação assistencial (MAYOL et al., 1987). 
Moreira e Goldani (2010), em análise recente sobre a situação da saúde da criança

em nosso meio, apontam para a necessidade de novas estratégias, relacionadas ao cuidado à criança e à formação de novos pediatras e pesquisadores, que deem conta do cuidado de um ser humano em crescimento e desenvolvimento, com todas as suas especificidades. Os autores alertam, ainda, que tais estratégias precisam ser implementadas nos serviços de saúde e no campo da produção de conhecimentos.

Especificamente em relação ao cuidado hospitalar, destacamos que a disponibilidade de informações, mesmo no campo da produção científica, sobre o perfil e as principais demandas das internaçōes pediátricas, ainda é escassa. Os resultados encontrados em pesquisas já realizadas são insuficientes e precisam ser aprimorados, no sentido de adequarmos as políticas de saúde para o novo perfil de adoecimento da criança e do adolescente.

Este artigo tem como objetivo apresentar o perfil clínico e demográfico das crianças internadas e as principais características em relação aos procedimentos diagnósticos e terapêuticos dessas internações, em quatro hospitais públicos federais no município de Rio de Janeiro.

\section{Material e métodos}

Foi realizado estudo retrospectivo das internações nos serviços de pediatria de quatro hospitais gerais que integram a rede hospitalar federal no Rio de Janeiro. Os hospitais foram codificados como H1 (28 leitos), H2 (25 leitos), H3 (24 leitos) e H4 (33 leitos).

Os quatro hospitais incluídos no estudo possuem centro de tratamento intensivo pediátrico, serviços de cirurgia pediátrica e um conjunto de outras especialidades pediátricas. Algumas características específicas desses serviços podem ser observadas entre os hospitais: dois deles ( $\mathrm{H} 1$ e o $\mathrm{H} 2)$ possuem serviço de emergência. $\mathrm{O}$ H1 é centro de referência em transplante hepático e renal pediátricos para todo o estado. Duas unidades atuam em oncologia pediátrica (H3 e H4).

Em 2008, ocorreram nesses serviços 2269 internações, de acordo com os dados do relatório anual dos hospitais. Desse universo foram selecionadas, de forma proporcional e aleatória, 170 internaçôes ocorridas nas enfermarias de pediatria no período de janeiro a dezembro de 2008 , de crianças desde o nascimento até 17 anos 11 meses e 29 dias. O tamanho da amostra foi calculado considerando uma prevalência de 50\%, uma margem de erro de $10 \%$ e um nível de confiança 
de $99 \%$. Dessa forma, estimava-se quantificar a variabilidade existente entre as internaçôes pediátricas a partir dos serviços considerados. Foram excluídas da amostra as internações que não completaram, no mínimo, 48 horas.

A coleta de dados foi realizada no período de setembro de 2009 a fevereiro de 2010. Para acesso aos prontuários, foi obtido previamente o consentimento das chefias médicas dos serviços de pediatria em cada unidade hospitalar.

Foi utilizado um instrumento elaborado especificamente para este estudo, construído em seis blocos de informações. No primeiro bloco foram incluídas as informaçōes demográficas do paciente tais como data de nascimento, sexo e local de moradia. O segundo bloco contém dados clínicos sobre o paciente quanto à existência de malformações congênitas, doenças genéticsa e outras condições clínicas com perfil de cronicidade definido de acordo com o critério encontrado no trabalho de Vargas, que considerou como crônica qualquer condição com duração maior que seis meses (VARGAS et al., 1994). O terceiro bloco reúne os dados referentes às internações - motivo, estado nutricional na internação, número de internações prévias e tempo de internação. A informação sobre o estado nutricional foi verificada no registro em prontuário para classificação do estado nutricional - "baixo peso", "risco nutricional", "sobrepeso", "desnutrição" e "peso adequado". O quarto e quinto blocos investigam especificamente as demandas relacionadas aos procedimentos diagnósticos e terapêuticos, objeto deste trabalho. O sexto bloco de dados inclui informações sobre solicitações de parecer técnico para a definição do diagnóstico.

Os dados sobre procedimentos diagnósticos e terapêuticos se referem à primeira solicitação de cada procedimento dentro das primeiras 72 horas de internação, período no qual a investigação diagnóstica e a estabilização do quadro clínico determinam uma demanda mais alta de utilização de tecnologias.

O presente estudo foi aprovado pelos Comitês de Ética de cada um dos hospitais após cumprir as exigências e padrões éticos das normas de pesquisa em seres humanos, conforme Resolução 196/96 do Conselho Nacional de Saúde (CNS), assegurando-se total sigilo das informações coletadas.

\section{Resultados}

Dentre as 170 internações selecionadas para investigação, 95 crianças (56\%) residiam no município do Rio de Janeiro e 75 (44\%), em outros municípios. 
A idade média das crianças internadas foi de 5.7 anos e a mediana, de 5.0, com alguma variação entre os quatro hospitais. $\mathrm{O} \mathrm{H} 1$ apresentou mediana da idade igual a 5.0; o H3 teve mediana de 5.5 e o H4 registrou 7.5. Destaca-se o H2 com mediana 1.0, inferior aos outros três hospitais.

Na distribuição da amostra por faixa etária, encontramos: 4.1\% de internações de crianças menores de um mês; $18.9 \%$ de internações de lactentes até 11 meses; $25.4 \%$ de crianças na faixa de 01 a cinco anos; $35.5 \%$ de crianças entre cinco e 12 anos; e 16.1\% de internaçôes de adolescentes com idade acima de 12 anos.

A tabela 1 apresenta as principais características das crianças estudadas em cada hospital, relativas à existência de malformações congênitas, doença genética ou de outras condiçôes clínicas com perfil de cronicidade.

Tabela 1. Proporção de internações de crianças com doença crônica nos serviços de pediatria de 4 hospitais públicos no MRJ

\begin{tabular}{|c|c|c|c|c|c|c|c|c|c|c|}
\hline \multirow[t]{2}{*}{ Variáveis } & \multicolumn{2}{|c|}{$\begin{array}{l}\text { Hospital } 1 \\
\mathbf{n}=37\end{array}$} & \multicolumn{2}{|c|}{$\begin{array}{c}\text { Hospital } 2 \\
n=33\end{array}$} & \multicolumn{2}{|c|}{$\begin{array}{c}\text { Hospital } 3 \\
n=50\end{array}$} & \multicolumn{2}{|c|}{$\begin{array}{c}\text { Hospital } 4 \\
n=50\end{array}$} & \multicolumn{2}{|c|}{$\begin{array}{c}\text { TOTAL } \\
\mathbf{n}=\mathbf{1 7 0}\end{array}$} \\
\hline & n & $\%$ & $\mathbf{n}$ & $\%$ & $\mathrm{n}$ & $\%$ & $\mathrm{n}$ & $\%$ & n & $\%$ \\
\hline $\begin{array}{l}\text { Malformação } \\
\text { congênita }\end{array}$ & 14 & 37,8 & 0 & & 2 & 4,0 & 3 & 6,0 & 19 & 11,2 \\
\hline Doença genética & 1 & 2,7 & 2 & 6,0 & 3 & 6,0 & 6 & 12,0 & 12 & 7,0 \\
\hline $\begin{array}{l}\text { Outras condiçôes } \\
\text { clínicas com perfil } \\
\text { de cronicidade }\end{array}$ & 4 & 10,8 & 5 & 15,1 & 22 & 44,0 & 19 & 38,0 & 50 & 29,4 \\
\hline $\begin{array}{l}\text { Doença crônica } \\
\text { (Total) }\end{array}$ & 19 & 51,3 & 7 & 21,2 & 27 & 54,0 & 28 & 56,0 & 81 & 47,6 \\
\hline
\end{tabular}

As doenças crônicas foram encontradas em 81 prontuários nos quatro hospitais e podem ser agrupadas da seguinte forma: Malformaçôes Congênitas $(\mathrm{n}=19)$; Doenças Genéticas $(\mathrm{n}=12)$; Outras condições clínicas com perfil de cronicidade $(\mathrm{n}=50)$.

Em relação às variáveis apresentadas na tabela 1, é importante destacar que 37,8\% das internaçôes no $\mathrm{H} 1$ eram de crianças com registro de alguma malformação congênita no prontuário, enquanto que no $\mathrm{H} 3$ apenas $4 \%$ dos prontuários estudados tinham esse registro. Para doença genética, o maior percentual encontrado foi de $12 \%$ no $\mathrm{H} 4$. 
Os dados relativos a outras condições clínicas com perfil de cronicidade permitiram constatar a frequência de $29,4 \%$ de agravos crônicos nas internações estudadas nos quatro hospitais, sendo que no $\mathrm{H} 3$ esse percentual chegou a $44 \%$. Nesse hospital, essas condições em sua maioria estavam relacionados a doenças onco-hematológicas.

No total da amostra pesquisada nas quatro unidades hospitalares, o conjunto de pacientes portadores de doença crônica representou 47.6\%. O somatório das malformações congênitas, doenças genéticas e condiçōes clínicas com perfil de cronicidade revelam resultados semelhantes nas internações pediátricas em três dos quatro hospitais estudados ( $\mathrm{H} 1, \mathrm{H} 2$ e H3). Nessas três unidades, encontramos frequências maiores do que 50\% para internações de pacientes com doença crônica. $\mathrm{O}$ H2 evidenciou um perfil diferente, com maior proporção de internações de lactentes, menor proporção de crianças com registro de doença crônica $(21.2 \%)$, menor frequência de pacientes com história de reinternações $(3,0 \%)$ e em acompanhamento ambulatorial (6,0\%), como pode ser visto na tabela 2 .

Em relação ao grupo de 81 pacientes portadores de doença crônica, encontramos 39 crianças (48.1\%) procedentes de outros municípios.

Os motivos de internação apresentaram grande variedade de casos no total da amostra e foram agrupados da seguinte forma: dengue $(n=36)$, procedimentos diagnósticos e terapêuticos e/ou intercorrências clínicas não infecciosas relacionadas à doença crônica não oncológica $(n=28)$, infecçôes em criança com doença crônica $(n=27)$, infecções respiratórias agudas $(n=20)$, procedimentos diagnósticos e terapêuticos e/ou intercorrências clínicas em crianças com doença oncológica $(n=19)$, primeira internação para investigação diagnóstica $(\mathrm{n}=11)$, gastroenterites agudas $(\mathrm{n}=8)$, infecçôes cutâneas $(\mathrm{n}=7)$, púrpuras $(n=3)$, traumas $(n=3)$ e síndrome nefrótica $(n=2)$. No grupo de infecções em crianças com doença crônica, predominaram as infecções respiratórias. Foram registradas ainda as seguintes causas de internação: encefalite por varicela zoster, meningite purulenta, doença de Kawasaki, infecção do trato urinário, infestação por áscaris e apendicite.

A tabela 2 apresenta outras características das internações estudadas. Para a variável "reinternação", o H3 e o H4 se destacaram, apresentando taxas de 46\% e $48 \%$ respectivamente. 
Tabela 2. Frequência de reinternação e acompanhamento ambulatorial, número médio de internações prévias e tempo médio de internação em pacientes internados nos serviços de pediatria de hospitais públicos no RJ

\begin{tabular}{lccccc}
\hline & $\begin{array}{c}\text { Hospital 1 } \\
\mathbf{n = 3 7}\end{array}$ & $\begin{array}{c}\text { Hospital 2 } \\
\mathbf{n}=33\end{array}$ & $\begin{array}{c}\text { Hospital 3 } \\
\mathbf{n}=50\end{array}$ & $\begin{array}{c}\text { Hospital 4 } \\
\mathbf{n}=50\end{array}$ & $\begin{array}{c}\text { Total } \\
\mathbf{n}=\mathbf{1 7 0}\end{array}$ \\
\hline Reinternação & $32,4 \%$ & $3,0 \%$ & $46,0 \%$ & $48,0 \%$ & $35,3 \%$ \\
$\begin{array}{l}\text { Internações prévias } \\
\text { (Média e Desvio Padrão) }\end{array}$ & $4,0(4,1)$ & $2,0(0,0)$ & $5,3(4,1)$ & $2,5(1,8)$ & $3,9(3,6)$ \\
$\begin{array}{l}\text { Tempo médio de } \\
\text { internação (Média } \\
\text { e Desvio Padrão) }\end{array}$ & $14,1(13,3)$ & $8,7(8,5)$ & $7,8(7,8)$ & $8,4(7,1)$ & $9,5(9,5)$ \\
$\begin{array}{l}\text { Acompanhamento } \\
\text { ambulatorial }\end{array}$ & $32,4 \%$ & $6,0 \%$ & $42,0 \%$ & $60,0 \%$ & $38,2 \%$ \\
\hline
\end{tabular}

No total da amostra, o grupo de pacientes com história de reinternação apresentou número de internações prévias variando entre uma e 17 internações no mesmo hospital.

Em relação à origem das internações, encontramos, no grupo estudado, $42 \%$ de internações que ocorreram a partir da emergência das unidades hospitalares estudadas, $13 \%$ encaminhadas do ambulatório dos próprios hospitais, 19\% por transferência de outros serviços, 10\% transferidas do CTI e 16\% de crianças que estavam em seus domicílios.

O estado nutricional das crianças internadas foi registrado em apenas 31 prontuários. Desse total, 06 apresentaram registro de "baixo peso" ou "risco nutricional", 04 apresentaram "sobrepeso", 04 evidenciaram quadro de "desnutrição", e 17 crianças foram referidas como "eutróficas". No restante dos prontuários ( $\mathrm{n}=139)$, não encontramos informações sobre o estado nutricional.

Os dados referentes aos procedimentos diagnósticos solicitados nas internações são apresentados na tabela 3. Nos quatro hospitais, o procedimento diagnóstico mais solicitado foi a radiografia simples com percentuais entre $42 \%$ (H3) e $72.7 \%$ $(\mathrm{H} 2)$. Cabe ressaltar que o procedimento diagnóstico raio-X simples se refere à realização do procedimento em qualquer localização. A ultrassonografia também se destaca como um dos procedimentos mais solicitados no $\mathrm{H} 4$ (40\%) e no H1 (37.8\%). Para hemocultura, encontramos a frequência de $42 \%$ no H4, enquanto 
que no $\mathrm{H} 1$ temos o registro de apenas 5.4\%. O mielograma e a punção lombar foram solicitados com maior frequência no $\mathrm{H} 3$ (10\% e 12\% respectivamente).

Tabela 3. Procedimentos diagnósticos solicitados nas primeiras 72 horas nos serviços de pediatria de 4 hospitais públicos no RJ

\begin{tabular}{|c|c|c|c|c|c|}
\hline & $\begin{array}{c}\text { Hospital } 1 \\
\mathbf{n}=37\end{array}$ & $\begin{array}{c}\text { Hospital } 2 \\
n=33\end{array}$ & $\begin{array}{c}\text { Hospital } 3 \\
n=50\end{array}$ & $\begin{array}{c}\text { Hospital } 4 \\
n=50\end{array}$ & $\begin{array}{c}\text { TOTAL } \\
\mathbf{n}=170\end{array}$ \\
\hline Raio X simples & $62,2 \%$ & $72,7 \%$ & $42,0 \%$ & $62,0 \%$ & $58,2 \%$ \\
\hline Ultrassonografia & $37,8 \%$ & $18,2 \%$ & $12,0 \%$ & $40,0 \%$ & $27,1 \%$ \\
\hline Hemocultura & $5,4 \%$ & $9,1 \%$ & $28,0 \%$ & $42,0 \%$ & $23,5 \%$ \\
\hline Exames sorológicos & $5,4 \%$ & $30,0 \%$ & $16,0 \%$ & $32,0 \%$ & $21,1 \%$ \\
\hline Ecocardiograma & $21,6 \%$ & $3,0 \%$ & $8,0 \%$ & $16,0 \%$ & $12,4 \%$ \\
\hline Gasometria arterial & $27,0 \%$ & $6,1 \%$ & $4,0 \%$ & $6,0 \%$ & $10,0 \%$ \\
\hline $\begin{array}{l}\text { Tomografia } \\
\text { computadorizada }\end{array}$ & $16,2 \%$ & $3,0 \%$ & $4,0 \%$ & $10,0 \%$ & $8,2 \%$ \\
\hline Urinocultura & $5,4 \%$ & $6,0 \%$ & $8,0 \%$ & $12,0 \%$ & $8,2 \%$ \\
\hline Punção lombar & - & - & $12,0 \%$ & $4,0 \%$ & $4,7 \%$ \\
\hline Mielograma & - & - & $10,0 \%$ & $2,0 \%$ & $3,5 \%$ \\
\hline Raio X contrastado & $5,4 \%$ & - & - & $2,0 \%$ & $1,8 \%$ \\
\hline Ressonância magnética & $2,7 \%$ & - & $4,0 \%$ & - & $1,8 \%$ \\
\hline Broncoscopia & - & $6,1 \%$ & - & - & $1,2 \%$ \\
\hline Teste do Suor & - & - & - & $4,0 \%$ & $1,2 \%$ \\
\hline Lavado gástrico & - & $3,0 \%$ & $2,0 \%$ & - & $1,2 \%$ \\
\hline
\end{tabular}

Os procedimentos terapêuticos mais observados nos registros dos prontuários estudados são apresentados na Tabela 4. A utilização de antibióticos mostrou-se elevada em todos os hospitais, variando de $54 \%$ (H3 e H4) a 70.3\% (H1). Da mesma forma, o acesso venoso periférico foi verificado na quase totalidade das internações (91\%). O acesso venoso profundo foi utilizado em $4,7 \%$ das internaçôes. 
internados nos serviços de pediatria de 4 hospitais públicos no RJ

\begin{tabular}{lccccc}
\hline & $\begin{array}{c}\text { Hospital 1 } \\
\mathbf{n}=37\end{array}$ & $\begin{array}{c}\text { Hospital 2 } \\
\mathbf{n}=33\end{array}$ & $\begin{array}{c}\text { Hospital 3 } \\
\mathbf{n}=50\end{array}$ & $\begin{array}{c}\text { Hospital 4 } \\
\mathbf{n}=50\end{array}$ & $\begin{array}{c}\text { TOTAL } \\
\mathbf{n}=170\end{array}$ \\
\hline Acesso venoso periférico & $81,0 \%$ & $94,0 \%$ & $92,0 \%$ & $96,0 \%$ & $91,0 \%$ \\
Antibioticoterapia & $70,3 \%$ & $60,6 \%$ & $54,0 \%$ & $54,0 \%$ & $58,0 \%$ \\
Balanço hídrico & $13,5 \%$ & $30,0 \%$ & $28,0 \%$ & $14,0 \%$ & $21,7 \%$ \\
Suporte de O2 & $29,7 \%$ & $27,0 \%$ & $6,0 \%$ & $12,0 \%$ & $17,0 \%$ \\
Transfusão de sangue & $8,0 \%$ & $6,0 \%$ & $20,0 \%$ & $12,0 \%$ & $12,4 \%$ \\
e hemoderivados & - & - & $16,0 \%$ & $8,0 \%$ & $7,0 \%$ \\
Quimioterapia & $10,8 \%$ & $3,0 \%$ & $4,0 \%$ & $2,0 \%$ & $4,7 \%$ \\
Acesso venoso profundo & & & & & \\
\hline
\end{tabular}

Para o procedimento "suporte de O2" foram registradas frequências semelhantes no H1 (29,7\%) e no H2 (27\%), e frequências menores nos outros dois hospitais (6\% para o $\mathrm{H} 3$ e 12\% para o $\mathrm{H} 4)$. Em relação à terapia transfusional, o H3 apresenta uma taxa de utilização (20\%) mais elevada em comparação aos outros três hospitais.

Encontramos em 11\% dos prontuários analisados relatos de procedimentos cirúrgicos, tais como drenagens de abscesso, biópsias, colocação e retirada de cateter para quimioterapia, confecção de fístula arterio-venosa, toracostomia com drenagem torácica e outros.

\section{Discussão}

O presente estudo possibilitou conhecer características das internações em enfermarias de pediatria ainda pouco apresentadas em publicaçōes brasileiras. A exclusão das internações com menos de 48 horas de duração possibilitou um grupo mais homogêneo em termos da efetiva necessidade de recursos do âmbito da internação, na medida em que possíveis indicações inadequadas de internação foram excluídas da análise.

$\mathrm{Na}$ amostra estudada, encontramos um percentual de $44 \%$ de pacientes oriundos de outros municípios, evidenciando-se uma tendência de deslocamento da população em direção aos serviços hospitalares dos grandes centros. Estudos 
realizados nas regiōes metropolitanas de São Paulo e Rio de Janeiro, de modo semelhante, descrevem o deslocamento da população para a região central em busca de atendimento (VECINA NETO; MALIK, 2007; CAMPOS et al., 2000). Essa tendência reafirma a necessidade de discussão sobre a estruturação de um sistema adequado de regionalização e de referência e contrarreferência para a atenção pediátrica, incluindo o suporte para acompanhamento e para o atendimento de intercorrências de menor complexidade em unidades secundárias mais próximas às suas residências dos pacientes com doenças crônicas. As questôes referentes às dificuldades de acesso e adesão à atenção especializada se tornam ainda mais evidentes em relação àqueles que residem em municípios distantes das famílias. Para esses pacientes, o trabalho em rede poderia possibilitar o tratamento em centros de saúde regionais, atenuando o impacto familiar produzido pela doença. (TOZIANO et al., 2004; SILVA, 2001; CAMPOS et al., 2000).

Em 42\% dos casos, as internaçoos foram de pacientes atendidos no serviço de emergência, sendo que as procedentes do ambulatório (13\%) correspondem a menos da metade das procedentes da emergência. Esse fato foi relatado em estudo que analisa as diferenças entre as internações ocorridas em um hospital regional de Minas Gerais, onde também foram encontradas mais internações provenientes de serviços de urgência e emergência, e menor número de internações eletivas, incluindo pediatria (CASTRO, 2001).

Foi registrada alta frequência de reinternações em três das quatro unidades estudadas. Em dois hospitais ( $\mathrm{H} 4$ e H3), encontramos praticamente a metade dos pacientes com história de internações prévias. Verificamos que, nesses hospitais, estão concentradas as internações de pacientes que são acompanhados (60\% no H4; $42 \%$ no H3; e $32.4 \%$ no H1) em ambulatórios especializados dessas unidades, o que nos permite inferir que, provavelmente, as reinternações ocorreram com frequência maior para os pacientes em tratamento prolongado. Em contraste com esse resultado, no $\mathrm{H} 2$, a proporção de pacientes acompanhados no ambulatório foi de 6\%, destacando-se que a maioria dos pacientes (97\%) apresentou uma única internação.

Em relação ao aspecto nutricional, o presente estudo destacou que a maioria das crianças não foi avaliada no momento da internação. Foi realizada, durante a pesquisa, uma busca minuciosa e detalhada, e encontraram-se apenas 31 prontuários (18,2\% das internações estudadas) com registro de avaliação 
nutricional. Considerando-se que a permanência hospitalar prolongada pode representar risco de desenvolvimento de desnutrição durante a hospitalização (KAC et al., 2000), é necessário que este seja rastreado precocemente para identificar os pacientes que necessitam de intervençôes nutricionais. Merece destaque a identificação de 14 internações de crianças que apresentaram alteração nutricional (seis com baixo peso, quatro com sobrepeso e quatro com sinais evidentes de desnutrição). Assim sendo, é extremamente preocupante que em 139 internações $(81,8 \%)$ não tenha sido encontrado registro de avaliação nutricional, entendido como um importante indicador da qualidade da assistência. Estudo desenvolvido por Duran (2001) enfatiza a necessidade de mensurar a situação nutricional de toda criança hospitalizada, independentemente de seu estado no momento da internação e do motivo.

O estudo permitiu identificar um percentual elevado (47.6\%) de agravos crônicos no grupo estudado, com três hospitais apresentando frequências maiores que $50 \%$. Merece ser destacado que esse resultado foi encontrado em serviços de pediatria em hospitais gerais que, embora considerados como referência para atendimento especializado, não são unidades de ensino e pesquisa ou centros de oncologia pediátrica ou de referência regional específica para determinado procedimento ou agravo, para os quais poderíamos prever um perfil de atendimento muito diferenciado. A exceção seria o $\mathrm{H} 1$ como referência estadual para transplante hepático ou renal em pediatria, eventos com baixa frequência no grupo estudado.

Além disso, deve ser considerado que as 36 internaçōes por dengue correspondem a um cenário específico (epidemia de 2008 ocorrida no estado do Rio de Janeiro), com reconhecida gravidade desse agravo entre crianças e adolescentes (BRASIL, 2009). Assim sendo, levando-se em conta de que se tratou de um fenômeno epidêmico, se analisarmos o conjunto restante de 134 prontuários estudados (excluindo-se os casos de dengue), obteremos uma proporção de $60,4 \%$ de crianças com doença crônica.

A proporção de agravos crônicos encontrada no presente estudo confirma resultados anteriores verificados por Vargas (1994), que estudou a frequência e o tipo de enfermidades crônicas em menores de 15 anos, tendo encontrado 35,2\% em um total de 469 internações pediátricas. O autor ressalta a importância de que os hospitais públicos estejam preparados para o manejo do paciente com esse perfil, que apresenta demandas específicas de recursos tecnológicos para o diagnóstico e tratamento. 
Deve ser destacado que, avaliando-se o conjunto de internações por razões ligadas às infecçôes em pacientes crônicos $(\mathrm{n}=27)$, intercorrências clínicas não infecciosas diretamente relacionadas à doença crônica $(n=28)$ e causas de internação para tratamento ou intercorrências da doença oncológica $(\mathrm{n}=19)$, encontramos um total de 74 motivos de internação (43,7\%).

Utilizando-se a mesma análise que fizemos anteriormente em relação à existência de doenças crônicas no conjunto das internaçōes, se excluirmos as internaçōes motivadas pela dengue, constatamos que fatores relacionados à doença crônica foram responsáveis por $55,2 \%$ das hospitalizações nos serviços de pediatria.

No que se refere aos casos de dengue, foram observadas três crianças portadoras de doença crônica. A infecção por dengue em crianças com doença crônica caracteriza bem a transição epidemiológica no contexto da saúde infantojuvenil. Os desafios no manejo das doenças crônicas na infância e adolescência se somam aos desafios ligados à ocorrência de doenças infecciosas emergentes ou não, características de situações com maiores dificuldades ambientais, sociais e do sistema de saúde. Nesse contexto, os riscos de maior gravidade dos casos de dengue em crianças com comorbidades são aspectos que devem merecer cuidadosa atenção, assim como em outras condições infecciosas com características epidêmicas, como é o caso da gripe por Influenza H1N1.

Os dados referentes aos procedimentos diagnósticos e terapêuticos solicitados nas primeiras 72 horas de internação revelam a frequência elevada de utilização de exames de imagem como radiografia simples e ultrassonografia. Foi encontrado percentual bem menos significativo para tomografia computadorizada (8.2\%) e ressonância magnética (1.8\%). Não foi objetivo deste trabalho analisar a pertinência da solicitação do procedimento diagnóstico ou terapêutico identificado nos prontuários. Entretanto, tendo em vista o perfil de morbidade já relatado para o grupo estudado, a proporção bem menor de solicitação de exames de imagem mais complexos pode ser parcialmente atribuída à dificuldade de acesso a esse procedimento.

Em relação aos outros exames, deve ser mencionada a maior proporção de exames como gasometria em dois dos quatro serviços de pediatria estudados $(\mathrm{H} 1$ e H2), indicando a possibilidade de maior gravidade das crianças internadas nessas unidades. Encontramos frequências maiores para mielograma e punção lombar no H3, unidade onde encontramos, na amostra estudada, maior 
proporção de internações por doenças onco-hematológicas. Nessa mesma unidade deparamo-nos com a maior proporção de transfusão de sangue ou hemoderivados, tendo sido observada em $20 \%$ das internaçôes. Esse achado reforça o perfil predominante de internações de doenças onco-hematológicas encontrado nessa unidade. De modo similar, a quimioterapia foi aplicada para $16 \%$ dos pacientes do $\mathrm{H} 3$, que possui serviço de onco-hematologia, sendo encontrada também em $8 \%$ das internações do H4.

Entre os exames sorológicos, destacamos as solicitações de sorologia para diagnóstico da dengue (14\%), em função da epidemia, que também contribuiu para a frequência de utilização do balanço hídrico encontrada nos pacientes incluídos no estudo. Em relação aos procedimentos terapêuticos, a utilização de antibióticos foi identificada para $58 \%$ dos pacientes internados, chegando a 70,3\% no $\mathrm{H} 1$.

Chamam atenção, neste estudo, as diferenças entre a utilização de antibióticos e solicitação de hemocultura, encontradas entre as quatro unidades. A utilização de antibióticos variou de 54\% no H3 e $\mathrm{H} 4$ chegando a 70,3\% no H1, enquanto a hemocultura apresentou resultados bem distintos e inferiores entre os hospitais (5,4\% no $\mathrm{H} 1,9.1 \%$ no $\mathrm{H} 2,28 \%$ no $\mathrm{H} 3$ e $42 \%$ no $\mathrm{H} 4$ ). Essa diferença foi acentuada no $\mathrm{H} 1$, onde observamos 70,3\% de utilização de antibióticos e 5,4\% de solicitações de hemocultura. Mesmo considerando que a indicação de hemocultura em internaçōes de enfermaria é definida pela gravidade do quadro infeccioso, essa discrepância chama atenção. Mais uma vez, o perfil de pacientes com agravos crônicos deve ser considerado como fator que exigiria maior rigor nas estratégias de utilização racional de antibióticos. Esse foi o quadro também encontrado em dois estudos sobre o uso de antimicrobianos em pediatria, os quais relatam que, na quase totalidade dos pacientes pediátricos hospitalizados, não foi encontrada nos prontuários médicos a informação sobre a realização de culturas ou antibiogramas (MEINERS et al., 2001; JOCK; SAKAE; DAL-BÓ, 2009).

A elevada utilização do acesso venoso periférico ( $91 \%$ dos prontuários estudados) é consequência de uma das principais indicações de internação em pediatria. Esse fato foi também relatado por Meiners (2001) que sugeriu o uso mais racional da via endovenosa, pois esse procedimento impóe ao paciente maior sofrimento e riscos, além de resultar em maior custo da atenção hospitalar.

Como observado em outros trabalhos, devemos destacar as dificuldades com a qualidade do preenchimento dos prontuários dos pacientes, os quais apresentaram 
anotaçōes incompletas, algumas vezes ilegíveis e codificadas (SCHOUT; NOVAES, 2007; MEINERS et al., 2001; FORMIGLI et al., 1997). Schout e Novaes (2007) ressaltam a importância dos registros clínicos para a qualidade da continuidade do cuidado aos pacientes e para a produção do conhecimento.

\section{Considerações finais}

O estudo identificou elevada frequência de crianças com doenças crônicas em enfermarias de serviços de pediatria, e apresentou os principais procedimentos diagnósticos e terapêuticos utilizados nesse grupo de internações.

Em três das quatro unidades hospitalares, as internaçôes de pacientes com doença crônica foram encontradas em mais da metade do grupo estudado. De forma semelhante, esses serviços também apresentaram elevado percentual de pacientes com histórico de reinternação.

Diante desse quadro, assim como observado em países que já passaram por essa transição, identificamos a necessidade de reorganização dos serviços pediátricos visando garantir estrutura, ambiência e processos de trabalho adequados para o manejo, tratamento e acompanhamento de pacientes com histórico de reinternações, internações prolongadas e necessidades de cuidado multidisciplinar.

Os diferentes aspectos que compõem o cuidado hospitalar destinado a esse grupo de pacientes devem ser incluídos na agenda de prioridades das políticas de saúde para a criança e adolescente no Brasil. Dentre eles, merecem ser citados: o efetivo dimensionamento das necessidades de recursos diagnósticos e terapêuticos com as especificidades inerentes a essas faixas etárias; a presença e a participação da família; a carga de trabalho dos profissionais envolvidos; as estratégias de redução do tempo de internação e desospitalização; e o acesso a ações de reabilitação.

Com a mesma importância, destacamos a necessidade de articulação das ações de saúde com as demais políticas públicas intersetoriais na perspectiva da garantia de direitos e a qualificação dos quadros técnicos e dos responsáveis pela tomada de decisão na atenção à saúde desses grupos. $\mathrm{O}$ atendimento integral de crianças e adolescentes que vivenciam a experiência do adoecimento crônico exige esse esforço. ${ }^{1}$ 


\section{Referências}

BRASIL. Ministério da Saúde. Informe Epidemiológico da Dengue. Análise de Situação e Tendências: semanas de 1 a 52 de 2009. Revisado. Disponível em: http://portal.saude.gov. br/portal/arquivos/pdf/informe_epidemiologico_semana_1a52_09_revisado.pdf Acesso em: 05 mar. 2010.

CAMPOS, T.P.; CARVALHO, M.S.; BARCELLOS, C.C. Mortalidade infantil no Rio de Janeiro, Brasil: áreas de risco e trajetória dos pacientes até os serviços de saúde. Rev Panam Salud Publica, v.8, n.3, p.164-171, 2000.

CASTRO, M.S.M. Comparação entre as internações de moradores de Betim no hospital público regional de Betim (HPRB) e em outros hospitais do Sistema Único de Saúde no estado de Minas Gerais. Cad Saúde Coletiva, v.9, n.1, p.53-68, 2001.

DURAN, P.; RAMOS, O. Modificacón en la situación nutricional durante la internación y fatores asociados. Arch argnt pediatr, v.99, n.5, p.405-413, 2001.

FORMIGLI, V.L.A. et al. Avaliação da atenção à saúde através da investigação de óbitos infantis. Cad Saúde Pública, v.12 (Supl. 2), p.33-41, 1996.

HEWSON, P.H. et al. A 12-month profile of community paediatric consultations in the Barwon region. J Paediatr Child Health, v.35, n.1, p.16-22, 1999.

JOBIN, R.; AERTS, D. Mortalidade infantil evitável e fatores associados em Porto Alegre, Rio Grande do Sul. Cad Saúde Pública, v.24, n.1, p.179-187, 2008.

JOCK, C.L.M.; SAKAE, T.M.; DAL-BÓ, K. Pneumonias na enfermaria de pediatria do Hospital Nossa Senhora da Conceição, em Tubarão-SC. Arq Cat Med, v.38, n.1, 2009.

$\mathrm{KAC}, \mathrm{G}$. et al. Length of stay is associated with incidence of in hospital malnutrition in a group of low-income Brazilian children. Salud Publica Mex, v.42, n.5, p.407-12, 2000.

LEJARRAGA, H. La atención pediátrica de pacientes crônicos, una prática necesaria. Arch argent pediatr, v.104, n.1, p.62-63, 2006.

MATLOW, A.G. et al. How can the principles of complexity science be applied to improve the coordination of care for complex pediatric patients? http://apps.who.int/whosis/data/ Search.jsp? countries=\%5bLocation\%5d. Acessado em: 15 fev 2010 .

\section{Nota}

${ }^{1}$ J.G. Duarte e M.A. de S.M. Gomes participaram da concepção do estudo e de todas as etapas da análise e discussão dos dados para a elaboração do artigo. S.C. Gomes e M.T. Pinto participaram da análise e discussão dos dados. 


\section{Abstract}

\section{Profile of pediatric services inpatients in the city of Rio de Janeiro: have we changed?}

Despite the changes observed in recent years in illness and mortality in childhood with a marked reduction in hospitalizations for infectious diseases, few studies exist that show the clinical and demographic profile of pediatric admissions in our midst. The study aimed to identify the characteristics and diagnostic and therapeutic procedures of pediatric admissions in hospitals in the public health system in the municipality of Rio de Janeiro. As to the method, a retrospective study conducted in four public hospitals in Rio de Janeiro, in a sample consisting of 170 hospital admissions in pediatric wards from January to December 2008. The information obtained showed the high frequency of children with chronic diseases $(47.6 \%)$ in all studied hospitals. The services also showed a high percentage of patients with a history of re-hospitalization (35.3\%). The analysis unit showed that among the four services studied, only one showed a lower proportion of children with chronic diseases and lower frequency of readmissions. In the other three hospitals, more than half of the group studied, the causes of hospitalization were found linked to chronic diseases. The results confirm the need for organization of pediatric services in order to be prepared, with appropriate structure and processes of care, for the management, treatment and monitoring of patients with chronic diseases.

> Key words: hospitalization; pediatric care; chronic illness in childhood. 